\title{
Violencia y crecimiento económico: un análisis empírico para Colombia ${ }^{1}$
}

\section{Violence and economic developmen: an empirical analysis of Colombia}

DOI: http://dx.doi.org/10.17981/econcuc.38.1.03

José Mauricio Gil León ${ }^{2}$

Wladimir Antonio Uribe Peñaranda ${ }^{3}$

\section{Resumen}

En el presente artículo se identifica la relación entre la violencia y el crecimiento económico en Colombia a través de una revisión de la literatura sobre estudios que explican el conflicto, considerando los estudios tradicionales y tendencias. Asimismo, se desarrolla un análisis de los antecedentes empíricos que explican el crecimiento económico por medio de la violencia y el conflicto armado. Los resultados se fundamentan en técnicas econométricas con las que se estima el efecto de las variables que se asocian a la violencia en el crecimiento económico, así como también determinan que en Colombia la violencia se explica por una debilidad institucional y afecta a la economía cuando los agentes sociales toman decisiones realizando actos criminales, como los secuestros, los homicidios y los delitos contra la propiedad. Se concluye, mediante los resultados de regresiones, que la tasa de variación de los secuestros, como variable asociada a la violencia, es la que tiene incidencia negativa en el crecimiento económico.

Palabras clave: actos de violencia; desarrollo económico; tasa de secuestros; conflicto armado.

Recibido: 23/1/2017 Devuelto para revisión: 30/3/17 Aceptado: 5/4/2017.

\footnotetext{
${ }^{1}$ Artículo de investigación científica

${ }^{2}$ Maestría en Economía. Especialista en Finanazas. Economista de la Universidad Pedagógica y Tecnológica de Colombia. Investigador júnior de la Universidad Pedagógica y Tecnológica de Colombia. mauricio8827@hotmail.com

${ }^{3}$ Cursante de la Maestría en Desarrollo Sustentable y Gestión Ambiental Universidad Distrital Francisco Jose de Caldas. Economista de la Universidad Pedagógica y Tecnológica de Colombia. wlado_1@hotmail.com
}

\author{
- The author; licensee Universidad de la Costa - CUC. \\ Económicas CUC vol. 38 no. 1, pp. 55-78. Enero - Junio, 2017 \\ Barranquilla. ISSN 0120-3932 Impreso, ISSN 2382-3860 Online
}




\begin{abstract}
The present article illustrates the interconnection between violence and the economic development in Colombia through a literatura review focused on previous researches and bias which have explained the mentioned conflicts from its empiric background with regard to the armed conflict. The obtained results are based on techniques of econometric analysis that explains violence as an institutional deficiency that affects the economic development when the social agents are inolved in criminal or illegal acts such as kidnappings, homicides and infractions against properties. It can be concluded that through the analisys of the preceeding results, the rate of divergence of kidnappings, was conceived as a relevant variable associated with violence which have had negative impacts on the the economic development of Colombia.
\end{abstract}

Keyword: acts of violence, economic development, kidnapping rate, armed conflict.

\title{
Introducción
}

Desde mediados del siglo XX el conflicto armado colombiano ha sufrido una serie de trasformaciones con hechos relevantes, como el inicio del plan Colombia -dado como un apoyo de EE.UU. para la lucha anti-narcóticos-, el recrudecimiento de las acciones militares de los diferentes bandos, la relación de la economía subterránea con actores paraestatales y los problemas asociados a éste, entre otros. De igual manera, en la sociedad colombiana hay una creciente percepción sobre actos criminales y violentos. Todos estos elementos mencionados, en parte, influyen en el comportamiento de los individuos y de los grupos sociales; reflejándose esto en las diferentes esferas sociales, como lo es la económica. 
Frente a un escenario de violencia y criminalidad los agentes sociales perciben la información del entorno y en base a ello toman decisiones, las cuales pueden determinar el rumbo de la economía, lo anterior se evidencia en diversos trabajos académicos que intentan retratar la dinámica económica bajo los factores de la criminalidad y la violencia. Echeverry, Salazar y Navas (2001) recurren a dos métodos diferentes para determinar la posible pérdida en el crecimiento económico que ha generado y genera el conflicto armado.

Con el primer método se busca observar el desempeño económico de un país con un conflicto armado contra países de la misma región, esto mediante la comparación de las tasas de crecimiento anuales en el período del conflicto armado. Como aspectos de interés se encontró que la mayoría de los países con conflicto armado tienen una tasa de crecimiento promedio menor durante el período de duración del conflicto armado, lo países con mayores pérdidas en las tasas de crecimiento fueron los que tuvieron conflictos armados de corta duración, al respecto se prevé que Colombia tiene un promedio de crecimiento por encima del promedio de la región.

La razón de por qué los conflictos armados de corto plazo son más costosos que los de largo plazo, los autores en mención, dan dos razones principalmente. La primera es que los agentes sociales en los conflictos armados de largo plazo, se adaptan a las dinámicas que implica la existencia del conflicto armado. La segunda razón, se halla en el método, con este se calcula la pérdida, vía tasa de crecimiento anual, y se ve que a medida que se alarga un conflicto, la pérdida del PIB se desacelera; no por ello hay que dejar de lado el factor acumulativo de estas pérdidas. Situación que se traduce en una desviación de la acumulación inicial de capital (Echeverry, Salazar y Navas, 2001)

En el segundo método los autores comparan el crecimiento económico promedio durante el conflicto con el crecimiento promedio de largo plazo; esto permite medir la desviación del país en conflicto con respecto a su tendencia de crecimiento, como consecuencia del impacto que genera el conflicto armado, por lo cual se considerar similitud en los resultados previamente mencionados. Frente a los conflictos armados de larga duración, sobre el choque permanente que este tipo de conflictos generan en la economía, se aclara que el "valor absoluto de la pérdida con respecto al valor de largo plazo puede verse sesgado por el cambio de este último, propiciado por la prolongación del conflicto en el tiempo" (Echeverry, Salazar y Navas, 2001, p. 101) 
Se considera, que en un intento por cuantificar la mayor pérdida de crecimiento por un conflicto armado, calcularon una aproximación del valor de la pérdida acumulada, como el resultado del producto de la pérdida promedio anual en la duración del conflicto. Señalan que “...de cualquier manera, un cálculo sofisticado de la pérdida de crecimiento debe contemplar, adicionalmente, los diversos canales a través de los cuales la violencia afecta el crecimiento en el tiempo, intensificando el efecto inicial". (Echeverry, Salazar y Navas, 2001, p. 101). Respecto a la leve pérdida del crecimiento de la economía colombiana con el conflicto armado, los autores explican que una posible razón para ello es el efecto "próspero" del narcotráfico sobre la dinámica económica.

En ese sentido, la pregunta de investigación es: ¿Qué relación existe entre los aspectos asociados a la violencia y el crecimiento económico? A partir del anterior cuestionamiento se va a analizar la literatura sobre violencia, y se realiza una estimación econométrica utilizando el segundo método expuesto por Echeverry, Salazar y Navas (2001), en donde se consideran datos anuales desde 1965 hasta 2013.

En definitiva, el documento se divide en cuatro secciones, incluyendo esta introducción como la primera. En la segunda sección se realiza una revisión de la literatura sobre el papel de la violencia en los diferentes aspectos sociales. En la tercera sección se considera la evidencia empírica, en donde se revisan algunos trabajos y se realiza una estimación econométrica utilizando datos entre 1965 y 2013. Finalmente, se realizan algunas conclusiones del documento.

\section{Fundamentos teóricos sobre la violencia}

Se puede hablar de diferentes formas de violencia, las cuales han ocurrido en distintos espacios temporales y de origen social distinto; incluso, dentro de las lógicas operativas del conflicto, los agentes violentos se han comportado de manera distinta en cada momento de la historia nacional (Tobar Torres, 2015). Gaitán (2001) plantea una discusión sobre la conceptualización de violencia, tomando como referencia el Informe de la Comisión de Estudios sobre la Violencia [CEV], convocada por la administración Barco y presentado en el año 1987. Pero Gaitán opta por la siguiente definición de violencia: "No todo hecho conflictivo es violencia, pero sí lo es el que causa daño físico” (Gaitán, 2001, p. 79). 
Otro concepto es el que toma la Organización Mundial de la Salud [OMS] en su informe del 2003. Este informe define la violencia como:

El uso intencional de la fuerza o el poder físico, de hecho o como amenaza, contra uno mismo, otra persona o un grupo o comunidad, que cause o tenga muchas probabilidades de causar lesiones, muerte, daños psicológicos, trastornos del desarrollo o privaciones (Organización Mundial de la Salud, 2003, p. 5)

Antes de definir la violencia para el presente documento es necesario aclarar que un hecho violento es un fenómeno social derivado de un conflicto ${ }^{4}$, pero no todo conflicto evoluciona hacia un hecho violento. Pero, ¿qué es conflicto? El conflicto se pude delimitar como aquellas situaciones en donde varios actores tienen intereses opuestos acerca de un tema en particular; y el desenlace de un conflicto puede significar que hayan desaparecido las causas que lo originaron o que no haya una resolución definitiva del conflicto, ya que este puede complejizarse debido a un cambio en los intereses y actitudes de los involucrados, lo que expresa como una evolución del conflicto (Esquivel Guerrero et al., 2009). La razón por la que la mayoría de conflictos no desembocan en actos violentos, se debe a que la sociedad misma ha desarrollado diversos mecanismos que le permitan solucionarlos. Una característica importante de estos arreglos sociales es que no son estáticos, y varían con el tiempo y de acuerdo a cada sociedad.

En la línea expuesta, los trabajos de Gaitán (2001) y la OMS (2003) señalan que la violencia es aquella situación en la que algunos individuos o grupos de personas no logran conciliar las diferencias de sus intereses coexistentes, y esta situación desemboca en el uso intencional de la fuerza, mediante los cuales se busca causar daños, como lesiones físicas o la misma muerte. Es necesario recalcar el vínculo que hay entre violencia y crimen, un vínculo casi implícito en la definición anterior ${ }^{5}$. En su mayoría, los actos violencia, son abordados desde la jurisprudencia, desde un marco policial y judicial, con el fin de castigar a quienes cometan tales actos. La mayoría de los actos de violencia son estudiados desde la perspectiva del crimen, pero no todos los actos criminales son de carácter violento. El presente documento

\footnotetext{
${ }^{4}$ A consideración del autor del presente trabajo, el trabajo CEV no plantean esta necesaria diferenciación entre conflicto y violencia, lo que les lleva a plantear todos tipo de violencias; esta falla la señalan inicialmente Gaitán y otros autores; en el presente trabajo se profundiza tal diferenciación con la inclusión necesaria del concepto de conflicto.

${ }^{5}$ Conceptualmente en diversos trabajos se toman como elementos de estudio independientes.
} 
no pone una línea divisoria entre ambos términos, tal y como lo proponen Montenegro y Posada (2001), debido a las características de la violencia.

El estudio de la violencia en Colombia ha sido amplio desde diversas ramas de las ciencias sociales. Todos estos estudios buscan entender la violencia, para ello, la mayoría recurren a buscar los determinantes de la violencia en Colombia. La revisión y el estudio de esta importante literatura permiten clasificarla en dos grupos principales ${ }^{6}$. El primer grupo se le clasifica como "Visiones Tradicionales"7, y el segundo grupo se le clasifica como "Nuevos Estudios" (Montenegro y Posada, 2001).

\section{Perspectiva de estudios tradicionales}

Dentro de este grupo se pueden mencionar dos trabajos sobresalientes sobre la violencia en Colombia. El primero de ellos, "La violencia en Colombia”, un trabajo de Guzmán, Fals Borda y Umaña (2014); el segundo trabajo es el presentado por la Comisión de Estudios sobre la Violencia [CEV] (1988).

Los estudios tradicionales llegan a unas afirmaciones sobre la violencia, que a manera general según Montenegro y Posada (2001) son las siguientes:

- La violencia ha sido un elemento constante y permanente de nuestra historia. Un fenómeno que se ha extendido a todos los ámbitos sociales, dando lugar a una cultura de la violencia.

- Existe continuidad entre los episodios violentos que han ocurrido a lo largo de la historia.

- Existen varias violencias relacionadas entre sí, que prácticamente recorren todos los espacios de la vida en el país.

\footnotetext{
${ }^{6}$ Muchos investigadores, especialmente desde la economía, resaltan esta diferenciación en los estudios nacionales sobre la violencia.

${ }^{7}$ De ahora en adelante esta categoría se simplificará como "estudios tradicionales".
} 
- La violencia en Colombia es una respuesta a una sociedad profundamente injusta y excluyente; específicamente, entre las causas se encuentran los altos niveles de pobreza, desigualdad económica y social, abandono del Estado y un problema con la endeble participación política de todos los agentes de la sociedad en Colombia.

Además, variables como la desigualdad, la educación y la presencia de grupos armados irregulares, están relacionadas con la violencia, ya que estos elementos, junto con una débil institucionalidad democrática, propician los espacios de violencia en Colombia; y en términos más específicos, sobre la relación entre violencia con la política, a pesar de que la democracia no es ficticia, en ésta si se han dado episodios de persecución a la oposición política y a la movilización social (Salas Salazar, 2015).

Dentro de las críticas a los estudios tradicionales se resalta el débil análisis empírico que estos estudios usan, ya que dichos análisis no pueden explicar de manera contundente ciertas hipótesis, como la de los mecanismos a través de los cuales la pobreza y la desigualdad inciden en la violencia y la criminalidad; por ejemplo, una comparación gráfica ${ }^{8}$ de países con sus tasas de pobreza, de desigualdad y de violencia ${ }^{9}$ se comprueba que países con niveles similares de pobreza y de desigualdad a los de Colombia, evidentemente, tienen menores niveles de homicidios (Montenegro y Posada, 2001).

\section{Perspectiva basada en nuevos estudios}

Los nuevos estudios recurren a novedosas metodologías, basadas principalmente en la recopilación y uso de más datos y variables, así como también de la aplicación de más pruebas estadísticas a los datos, para llegar a un análisis estadístico más riguroso.

Por ejemplo, frecuentemente se asegura que la desigualdad y la pobreza generan violencia, pero Gómez (2001) afirma que en el caso colombiano, una revisión de las cifras visibiliza el

\footnotetext{
${ }^{8}$ Las gráficas se pueden encontrar en el libro "La Violencia en Colombia” (2001), de Montenegro y Posada, en las páginas 15 y 16 .

${ }^{9}$ La violencia es representada por el parámetro de la tasa de homicidios por cien mil habitantes.
} 
aumento de los niveles de violencia durante los últimos 20 años ${ }^{10}$ ha sido casi similar a la desigualdad económica, acentuándose más la brecha entre ricos y pobres; esto se ve reflejado en los niveles de bienestar de las áreas urbanas y rurales; y a pesar de la alta correlación entre el aumento de los niveles de violencia y de desigualdad, no se puede afirmar que ésta última sea la causa de la primera, y, esto se debe a que hay países con niveles de desigualdad similares a los de Colombia, pero sin los niveles de violencia de ésta; sin embargo, hay que tener en cuenta que en esos países no existen grupos paraestatales como los de Colombia, ni un contexto histórico como es el colombiano. El fuerte desarrollo de la violencia en áreas rurales no sólo genera fuertes afectaciones en la economía de dichas áreas, sino que también ha generado y genera un desplazamiento forzado hacia las ciudades, con todas las consecuencias económicas y sociales de este fenómeno. Lo anterior permite plantear una hipótesis contraria: la violencia generadora de la desigualdad (Gómez, 2001).

Gómez (2001) sostiene que la insatisfacción producida por la desigualdad es generadora de violencia, siendo ésta respaldada por un discurso político. Esta hipótesis sobre la violencia requiere una explicación más elaborada, debido a que la teoría de la privación relativa ${ }^{11}$ tiene una escasa validez empírica; ello ha motivado a los investigadores a formular distintas hipótesis, y dentro de estas se encuentra la de la "escuela de movilización de recursos".

En este enfoque no solo se descartan las relaciones postuladas por las hipótesis de privación relativa, sino que, además, se argumenta que la variable explicativa central debe encontrarse en la medida en que los grupos disidentes son capaces de adquirir el control de los recursos necesarios para desarrollar una organización fuerte y efectiva con el propósito de obtener bienes colectivos. (Gómez, 2001, pp. 45-46)

Entonces, Gómez (2001) asegura que atribuir a la inequidad como la causa del conflicto, es una simplificación engañadora que oculta el marco sobre el cual se desenvuelve la violencia en Colombia, y de una manera más amplia plantea que la desigualdad económica cuando se junta con otros elementos, como los son un régimen político semidemocrático y oportunidades de alzarse en armas, se adecuan las condiciones para buscar objetivos políticos mediante el uso de la violencia.

\footnotetext{
${ }^{10}$ Hay que tener en cuenta que los 20 años en mención son de las décadas de los ochentas y noventas, ya que el libro fue publicado hacía el 2001.

${ }^{11}$ Teoría que afirma que la desigualdad y la pobreza generan insatisfacción en los individuos.
} 
En un estudio realizado por Collier y Hoeffler (2001), (Citado en Montenegro y Posada, 2001), encontraron que la rebelión es más probable en aquellas economías que tienen una alta dependencia de las exportaciones de bienes primarios. Según Collier y Hoeffler la razón radica en que estos bienes son fáciles de capturar por los actores violentos, y esto les permite financiar sus actividades violentas.

\section{A lo cual Montenegro y Posada afirman (2001):}

La relación entre violencia y crecimiento se ha hecho evidente en las llamadas economías de frontera, en los escenarios de acelerada prosperidad en regiones apartadas a causa del desarrollo súbito e intenso de productos primarios (oro, petróleo, esmeraldas, banano, coca), que reciben migraciones masivas e incorporan nuevas formas de producción que generan sustanciales ganancias (Montenegro y Posada, 2001, p.4)

Siendo así, para estos estudios, los actores armados actúan muy por encima de razones políticas, y más bien estos actores actúan bajo cierta racionalidad económica, en la cual siempre buscan maximizar sus beneficios, además, dicha maximización está relacionada directamente por la oferta estatal concerniente con la justicia y la seguridad (Salas Salazar, 2015).

La rebelión en sí misma, independiente de las razones políticas, genera escenarios de violencia. En la mayoría de los casos de violencia persisten diversos crímenes, por esta razón es importante tener en cuenta el rol del sistema judicial del país, o cualquier escenario del mundo en el que se desarrolle un escenario violento. Por esta razón, algunos investigadores centran su estudio en las fallas del sistema judicial y policial. Un ejemplo de este enfoque lo hace Rubio (1998) en su libro “Crimen e impunidad: Precisiones sobre la violencia.”; en éste evalúa la evolución del sistema judicial colombiano, con el fin de encontrar aquellos elementos que indiquen los fallos del sistema judicial que inciden en el aumento del crimen y la violencia.

Rubio (1998) encuentra que las diferentes reformas a la justicia afectaron los criterios para investigar los actos criminales; inicialmente estas reformas se concibieron para que los investigadores judiciales y demás miembros del sistema judicial les dieran una mayor prioridad a aquellos crímenes "ameritaban" ser investigados; esto "le otorgó una patente de corso al crimen organizado", que le fortaleció, e hizo que éste se incentivara a realizar prácticas más violentas. Tales reformas al sistema judicial colombiano, paulatinamente, fueron "dele- 
gando" a los mismos ciudadanos la tarea de aclarar los crímenes; con ello se fue expandiendo la información a las diferentes esferas de la sociedad sobre del debilitamiento del sistema judicial, dando a lugar para que muchos ciudadanos buscarán hacer justicia por sus propias manos, que a la final se tradujo en más violencia. Estas fallas del sistemas judicial y policial, se interpreta como unos de los causantes de la violencia en el país.

En un trabajo anterior, Rubio (1997) menciona los escenarios en que el Estado abandona (o su presencia es muy débil) un territorio o un mercado, y de que esos escenarios surgen las instituciones paraestatales, que buscaban y buscan apoderarse de los vacíos de poder del Estado, logrando ello mediante el establecimiento de sus reglas de juego.

La experiencia colombiana es rica en ejemplos de vacíos de poder de dónde surgieron paraestados privados que mediante la acumulación del poder derivado del uso exitoso de la fuerza terminaron cogobernando. Los casos más prominentes, más no los únicos en Colombia, serían la guerrilla, los narcotraficantes y los grupos paramilitares. Aunque con objetivos iniciales radicalmente distintos estas organizaciones se hicieron exitosas básicamente con dos elementos. Un amplio conocimiento de las instituciones, formales e informales, colombianas que les permitió aprovechar todas las fisuras del sistema -detectar los vacíos de estado- y, sobretodo, el uso permanente y sistemático de la violencia (Rubio, 1997, p.4).

Ahora, Sánchez, Díaz y Formisano (2003), señalan que uno de los aspectos más importantes a la hora de estudiar la violencia en Colombia, es el análisis de la dinámica de expansión y difusión de grupos armados. El análisis espacial puede determinar los patrones de difusión de la violencia y de actividades criminales pertenecientes a los grupos armados, en un modelo de contagio.

Por ejemplo, una organización ilegal que compite por el dominio de un mismo territorio puede desatar un conjunto de ataques y represalias por parte de otra que lucha por el mismo territorio. Los ataques y represalias pueden afectar a individuos o poblaciones no participantes, llevando a un incremento generalizado de la violencia (Sánchez, Díaz y Formisano, 2003, p. 22).

Resaltando el punto de vista geográfico del estudio de la violencia, se puede evidenciar ciertas lógicas y estrategias de los actores armados en tres espacios geográficos: nacional, regional y local. La territorialidad ejercida en dichos espacios se exhibe a través de distintas estrategias, mediante las cuales buscan mantener dichos espacios, de acuerdo al alcance de su poder, pero, a final de cuentas lo que se busca mantener o ganas son espacios económicos 
y políticos; es necesario señalar que los objetivos de estos tres espacios territoriales, en la mayoría de los casos, se relacionan (Salas Salazar, 2010).

De esta manera, se puede inferir que cuando son altos los indicadores de violencia, como es el de asesinatos, es porqué hay una disputa territorial entre diversos grupos armados. Cuando los niveles de asesinatos son bajos, significa que hay dominación de un grupo armado, ya sea de las fuerzas militares del Estado o de un grupo paraestatal. La dominación de un grupo armado, especialmente de los paraestatales, no significa que estén solucionados los conflictos que desembocaron en la violencia, de hecho, pueden traducirse en el aumento de los niveles de otros indicadores violentos, como por ejemplo el del desplazamiento forzado. Este posible aumento en los distintos indicadores al de asesinatos, podría significar que los grupos paraestatales en sus intentos por mantener el control territorial recurren a cometer diversos actos criminales.

Ahora, en una investigación realizada por Sánchez y Núñez (2001), se estiman diversos modelos econométricos y seleccionan algunas variables que permiten corroborar ciertas hipótesis, como la de la exclusión política, ya que ésta es propuesta en los estudios tradicionales como una de las razones para la violencia en Colombia; para verificar ésta hipótesis recurren a los datos de la participación electoral, ésta tomada como parámetro de la participación política. De acuerdo con el resultado el nivel de participación política tiene un papel bastante reducido en la explicación de la violencia (Sánchez y Núñez, 2001). Tomar el nivel de participación electoral como único parámetro para medir la exclusión política es un error, pues ya que este parámetro no tiene en cuenta afectaciones comunes de la participación política en Colombia, como lo son: la compra de votos, la intimidación electoral por parte de grupos armados para favorecer a un determinado candidato, etc.

El conflicto armado colombiano ha evolucionado desde sus primeros brotes, los cuales se evidencian hacia finales de los años cincuenta. Hasta el presente, el actor más persistente ha sido el Estado colombiano; también han desaparecido del escenario diferentes grupos paraestatales, dentro de los grupos desaparecidos se pueden mencionar a las guerrillas liberales o el grupo denominado "Movimiento 19 de abril" [M19], el grupo de las FARC (Fuerzas Armadas Revolucionarias de Colombia), con el que se finalizó en el 2017 el acuerdo de paz, y grupos que persisten en la movilización como: el Ejército de Liberación Nacional 
[ELN] y algunos grupos Paramilitares ${ }^{12}$. A pesar que los grupos armados han mantenido unos lineamientos en sus discursos políticos, las estrategias para mantenerse en el escenario han cambiado con el tiempo, y por lo tanto han cambiado algunos de sus objetivos o han adoptado unos nuevos. Una prueba de los cambios de estrategias y objetivos en los grupos paraestatales fue la aparición del fenómeno del narcotráfico, siendo este fenómeno un gran punto de inflexión en el conflicto armado.

Por su parte, Estrada (2009) recurre a una explicación más dinámica sobre el conflicto armado en Colombia, basado principalmente en la teoría de juegos. El análisis de la evolución del conflicto de Estrada (2009) se centra en cómo la evolución del conflicto se vio influenciada por el mercado potencial de la protección y seguridad privada, determinado por la geografía nacional. Tal mercado fue reforzado por el endeble alcance del Estado en temas de seguridad y justicia, principalmente en aquellas zonas de la periferia. Las organizaciones paraestatales y sus miembros actúan bajo su propia racionalidad para conseguir sus objetivos, tales conductas para muchas personas no pertenecientes a estos grupos son irracionales y sorprendentes.

Las nuevas guerras no presentan una ruptura total con las estrategias del conflicto de los años sesenta, pero incorporan aspectos, que como el narcotráfico y el contrabando, hace que la explicación de las nuevas guerras sea algo más complejas. Además, "en principio, bajo mecanismos sutiles de oferta de protección a bajos costos, y luego empleando la intimidación, los grupos paramilitares e insurgentes lograron penetrar la política local, y en algunos casos su intervención excedía relaciones sociales básicas” (Estrada, 2009, p. 163).

Estrada (2009) hace hincapié en que los contextos económicos y sociales deplorables dan significativos motivos para que una persona decida pertenecer a los grupos paraestatales, llegando incluso en muchos casos a desaparecer el mismo discurso político de éstas agrupaciones; a partir de ello las situaciones de pobreza, desigualdad, desempleo, corrupción y asimetrías regionales cobran importancia para el análisis de la violencia en Colombia. Estrada (2009) rechaza la idea de que la protección y la seguridad que ofrecen los grupos paraestatales, por ejemplo a narcotraficantes u otros mercados ilegales, son independien-

\footnotetext{
${ }^{12}$ Aunque se desmovilizaron en nombre de las Autodefensas Unidas de Colombia [AUC], muchos miembros volvieron a formar nuevos grupos paramilitares.
} 
tes de los objetivos del conflicto armado; en tanto, "las relaciones entre los carteles con el paramilitarismo o entre los carteles y la guerrilla, tanto como las relaciones que tienen organizaciones criminales con eslabones menores de la cadena, concuerdan con numerosos objetivos" (Estrada, 2009, p. 172ᄀרᄀ).

Por otro lado, en los estudios económicos sobre la violencia, unos se centran en los costos que conlleva la violencia, más que centrarse en los determinantes del conflicto y la dinámica de éste. El análisis de los costos se centra en determinar cómo éste afecta el crecimiento económico, en las regiones o el país mismo. Según Restrepo (2009) el conflicto armado lleva a una reasignación de recursos hacia actividades que probablemente sean más improductivas; por ejemplo, en vez de invertir más en investigación y desarrollo de productos industriales, capital humano o infraestructura, estos recursos pueden ser destinados a un mayor gasto militar, lo cual implica un menor crecimiento económico.

\section{Antecedentes basados desde una visión empírica}

El análisis empírico se ha fundamentado en utilizar los modelos de crecimiento económico más relevantes, donde diversos autores recurren a modificar estos modelos, con el fin de incluir la violencia como variable influyente en el crecimiento económico. Por tanto, "la razón para ello se debe a que un análisis tradicional basado en los tres factores es insuficiente para explicar las divergencias en el desempeño económico de los diferentes países" (Molina y Hurtado, 2012, p. 3)

Molina y Hurtado (2012) recurren a modificar el modelo de Solow. La modificación se da haciendo la inclusión de la violencia, y considera que ésta afecta la acumulación de factores productivos, vía destrucción o reasignación ${ }^{13}$ de recursos. Además, hay que tener en cuenta los costos en los sistemas judiciales ${ }^{14}$, y también los daños en el tejido social. Cuando el tejido social se ve afectado repercute directamente en la formación de capital humano.

\footnotetext{
${ }^{13}$ La reasignación de recursos se traducen en la búsqueda de mejoras en la seguridad privada, esto es para solventar los problemas de inseguridad.

${ }^{14}$ Apalancando la debilidad institucional.
} 
Debido a la dificultad que implica cuantificar la violencia, Molina y Hurtado (2011) recurren a medirla mediante el cálculo de la tasa de homicidios por 100 mil habitantes. Dentro de los resultados se encontró que la violencia a largo plazo impacta negativamente el PIB a razón de un $-5.3 \%$. Las conclusiones dan por hecho que un mejoramiento en los niveles educativos y la reducción de los homicidios contribuyen positivamente al crecimiento económico del país.

Por otro lado, Hofstetter (1998) intenta probar a nivel teórico cómo la violencia afecta la acumulación de factores y el crecimiento económico, tanto en el corto pazo como en el largo plazo, recurriendo a dos modelos. Uno de crecimiento exógeno y otro de crecimiento endógeno; ambos con sus respectivas modificaciones para medir el impacto de la violencia. Hofstetter (1998) reconoce que la violencia genera efectos nocivos sobre los factores de producción, en base a ello justifica parte de su trabajo. Inicialmente, Hofstetter (1998) grafica la relación entre la tasa de homicidios por cien mil habitantes, contra, el crecimiento del PIB. El resultado que obtiene es que hay una relación negativa entre estas dos variables, lo cual indica que la violencia tiene un efecto recesivo sobre el crecimiento económico; además, resalta los efectos que generaría una desaceleración del PIB sobre la actividad criminal violenta, en el sentido de estimularla.

Para el modelo de crecimiento exógeno, Hofstetter (1998) recuerda que para incrementar el producto de una economía bajo el análisis neoclásico, es necesario aumentar el stock de capital. Para lo anterior debe existir mayor inversión, la cual depende del nivel de ahorro. Teniendo en cuenta la depreciación que sufre el capital a lo largo del tiempo, se resalta en este modelo el efecto de depreciación que tiene la violencia sobre el capital, debido al efecto destructor de la violencia. La violencia es tomada como una variable de depreciación adicional. El problema de esta variación del modelo presentado, según el mismo autor, es que no tiene en cuenta los efectos negativos que tiene la violencia sobre el capital humano (Hofstetter, 1998).

El problema del modelo exógeno de crecimiento, Hofstetter (1998), busca solucionarlo con el desarrollo de un modelo de crecimiento endógeno, en el cual trata de medir principalmente los efectos de la violencia sobre el capital humano, esto a razón de que los agentes ven afectadas sus decisiones de formación de capital humano, por un elemento como es la violencia, que se traduce en menores tasas de crecimiento en la productividad, vía afectación del capital humano. 
Ahora, Villa, Moscoso y Restrepo (2013) desarrollan un modelo de Solow-Swan modificado, en el cual estudian las consecuencias del conflicto armado y del crimen organizado sobre el ingreso de la economía. Su estudio se centra a nivel departamental. Encuentran que la elasticidad del PIB departamental con respecto a las variables de conflicto armado y crimen organizado, se encuentra en promedio entre el -0.04 y el -0.36 , respectivamente. De igual manera, advierten que un aumento del 10\% del conflicto armado o del crimen organizado, afecta negativamente en $1 \%$ el PIB departamental.

En una investigación realizada en México, a través técnicas econométricas se pretendió arrojar evidencia empírica sobre la relación entre el crecimiento económico regional (a nivel de los estados de México) y los niveles de delincuencia y criminalidad; el análisis econométrico se realiza mediante dos modelos econométricos: un modelo base y otro alternativo. Desde el punto de vista de las variables económicas, ambos modelos comparten las mismas variables, pero, la variable dependiente en ambos modelos es el crecimiento económico medido con el PIB per cápita. La distinción entre los dos modelos se evidencia en las variables de criminalidad y violencia, y, el modelo interesante es el alternativo, ya que en éste se investiga el efecto de las variables criminales como lo son: robos, lesiones dolosas, homicidios culposos, violaciones como delitos sexuales y los secuestros (González Andrade, 2014).

Dicha investigación resalta, dentro de sus resultados del modelo alternativo, que estos indican que los delitos de alto impacto, tales como homicidios, robos, y lesiones dolosas ejercen un efecto negativo sobre el crecimiento económico regional, por lo que se encuentra evidencia estadística que la delincuencia y criminalidad del tipo común posee cierto impacto negativo en el crecimiento económico regional, y frente al modelo alternativo, también la incidencia de algunos de los delitos considerados de alto impacto, tales como homicidios, robos y lesiones dolosas ejercen un efecto negativo sobre el crecimiento económico regional (González Andrade, 2014).

\section{Metodología}

Debido a la falta de continuidad de datos de una misma fuente, se utilizaron distintas fuentes para construir cada indicador, eventualidad que se presentó en cada uno de los indicadores. Los indicadores a analizar se presentan en tasas de crecimiento anuales de 1965 a 2013, esto con el fin de observar la dinámica de las variables. 
Variables:

- Tasa de crecimiento anual de homicidio: La primera fuente corresponde al Instituto de Medicina Legal, que comprende los años de 1965 al 2001; la segunda fuente que corresponde al Ministerio de Defensa, comprende los años del 2002 al 2013. Una vez empalmadas las cifras de homicidios, se procedió a calcular la Tasa de Crecimiento Anual de Homicidios.

- Tasa de crecimiento anual de secuestro: La primera serie que corresponde el período de 1965 al 2001, las fuentes principales son la Policía Nacional, y las organizaciones de País Libre y Fondelibertad. La segunda serie, que corresponde al período de 2002 al 2013, su fuente principal es el Ministerio de Defensa. Ante los diferentes tipos de secuestros, se ha recurrido a dos categorías para retratar ítem del secuestro, estas categorías son: secuestro simple y el secuestro extorsivo. Una vez empalmadas las cifras de secuestros, se procedió a calcular la Tasa de Crecimiento Anual de Secuestros.

- Tasa de crecimiento anual de delitos contra la propiedad: La primera fuente corresponde a la Policía Nacional, y ésta comprende los años de 1965 al 2001; la segunda fuente que corresponde al Ministerio de Defensa, bajo la categoría de "Protección del patrimonio económico" pero que corresponde al rubro de "Delitos Contra la Propiedad"; los datos del Ministerio de Defensa comprenden los años del 2002 al 2013. Una vez empalmadas las cifras de Delitos Contra la Propiedad, se procedió a calcular la Tasa de Crecimiento Anual de Delitos Contra la Propiedad.

- Tasa de crecimiento anual del Producto Interno Bruto: La primera fuente corresponde al Grupo de Estudios del Crecimiento Económico Colombiano, GRECO, del Banco de la República; la serie de esta fuente corresponde al período de 1965 a 1990. La segunda fuente corresponde a la CEPAL, cuya serie corresponde al período de 1991 al 2013, la cual presenta directamente la Tasa de Crecimiento Anual del PIB para el periodo en mención. Una vez revisadas las cifras de cada una de las fuentes, se procedió a calcular la Tasa de Crecimiento Anual del PIB de la primera fuente; posteriormente de ello, se empalmaron las tasas de crecimiento anuales. 
- Tasa de crecimiento anual de la formación bruta de capital fijo: La primera fuente corresponde al UMACRO-DNP; la serie de esta fuente corresponde al período de 1965 al 2000. La segunda fuente corresponde al DANE, cuya serie corresponde al período de 2001 al 2013. Debido a los diferentes rubros que componen la cuenta de FBKF, se ha optado por los rubros de "Construcción y vivienda", y, "Maquinaria y equipo"; esto a razón de que son los rubros que presentan continuidad para el período de estudio. Una vez ordenadas las cifras se procedió a calcular la Tasa de Crecimiento Anual de Delitos Contra la Propiedad, para posteriormente empalmar tales tasas de crecimiento.

- Tasa de Crecimiento Anual de la Población Económicamente Activa (PEA): La primera fuente corresponde al DANE; la serie de esta fuente corresponde al período de 1965 al 2000. La segunda fuente corresponde al CELADE - División de Población de la CEPAL, cuya serie corresponde al período de 2000 al 2013 . Una vez ordenadas las cifras se procedió a calcular la Tasa de Crecimiento Anual de la PEA, para posteriormente empalmar tales tasas de crecimiento.

En el gráfico 1, se observa la dinámica de la tasa de crecimiento de las variables asociadas a la violencia y el crimen. Se puede observar que entre 1965 y 2013 lo que tuvo mayor fluctuación fue la tasa de secuestros, observando que los mayores incrementos ocurrieron en los periodos 1985-1990 y 1997-2000. La tasa de homicidios le sigue en variación, y se identifica que los periodos en donde creció en mayor número los secuestros también los hizo la tasa de homicidios. Por último, la variación de los delitos en contra de la propiedad no ha tenido fuerte fluctuación, pero lo que se evidencia es que antes de 1985 tenía un comportamiento similar a las otras dos variables, pero después hubo una adaptación de las fuerzas de seguridad al conflicto, lo cual condujo a que el crecimiento de estos delitos no cambiara considerablemente. Un aspecto a destacar es que después de 2003 la tasa de secuestros cayó y los homicidios no aumentaron, pero los delitos contra la propiedad sí lo hicieron, por lo que es posible deducir que la política de "seguridad democrática" atacó las principales causas de homicidios y secuestros, pero no lo hizo de la misma forma con los actos criminales comunes (robos, atracos, etc.). 


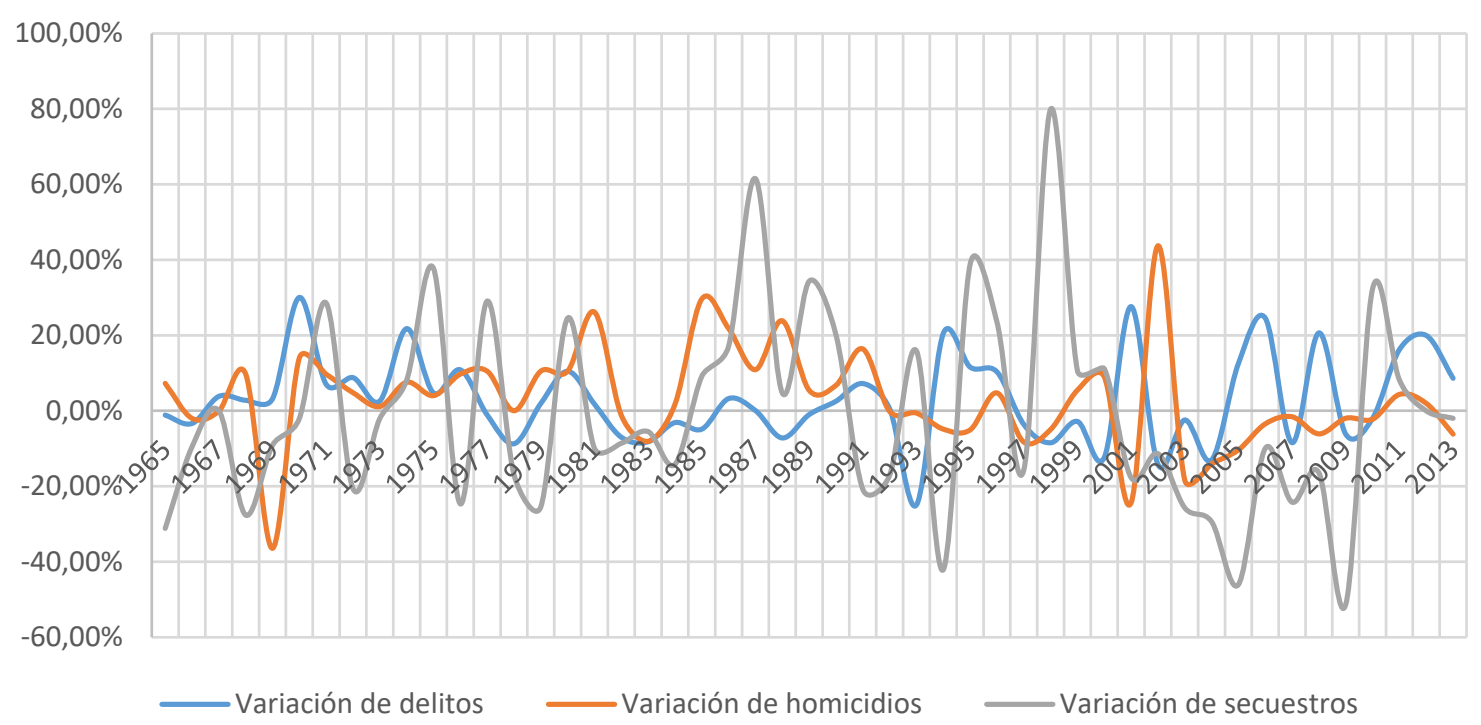

Gráfico 1. Comportamiento de la tasa de crecimiento de los delitos, homicidios y secuestros Fuente: datos de ministerio de defensa, policía nacional, país libre y fondelibertad

Las variables señaladas se toman para explicar la tasa de crecimiento económico, y se toma como base teórica al modelo de crecimiento de Solow modificado, con el fin de introducir las proxys de violencia y criminalidad como son: la tasa de crecimiento anual de homicidios, tasa de crecimiento anual de daños contra la propiedad, y la tasa de crecimiento anual de secuestros.

El modelo econométrico a estimar es el siguiente:

$$
g y_{t}=\hat{\beta}_{0}+\hat{\beta}_{1} g k_{t}+\hat{\beta}_{2} g l_{t}+\hat{\beta}_{3} t h_{t}+\hat{\beta}_{4} t s_{t}+\hat{\beta}_{5} t d_{t}+\mu_{t}
$$

La variable dependiente corresponde a la tasa de crecimiento anual del PIB $\left(g y_{t}\right)$ de la economía. Dentro de las variables independientes del modelo se encuentra: La tasa de crecimiento de la Formación Bruta de Capital Fijo de la economía colombiana $\left(g k_{t}\right)$ es la forma de identificar la acumulación de capital físico. La tasa de crecimiento de la Población Económicamente Activa $\left(g l_{t}\right)$ representa la manera cómo va creciendo el factor trabajo. Las variables de conflicto 
y crimen son la tasa de crecimiento anual de homicidios ((th), la tasa de crecimiento anual de secuestros $\left(t s_{t}\right)$, y la tasa de crecimiento anual de delitos contra la propiedad $\left(t d_{t}\right)$. Se espera que las variables relacionadas con la violencia y crímenes incidan en el crecimiento económico. Finalmente, $\mu_{t}$ representa los residuos, es decir, las diferencias entre los valores observados y estimados del crecimiento de la economía colombiana, donde además se encuentran aquellas variables que no pudieron ser incluidas en el modelo por razones metodológicas.

\section{Resultados}

El modelo econométrico se estima con el método de Mínimos Cuadrados Ordinarios (MCO), y se refleja en la tabla 1.

Tabla 1

Determinantes de la tasa de crecimiento del PIB de Colombia

Variable Dependiente: Tasa de crecimiento del PIB

Muestra: 1965-2013

Mínimos Cuadrados Ordinarios (MCO)

Errores estándar consistentes con heterocedasticidad

Errores estándar ()

\begin{tabular}{lccc}
\hline \multicolumn{1}{c}{ Independientes } & {$[1]$} & {$[2]$} & {$[3]$} \\
\hline Constante & -0.00812 & -0.00073 & -0.00752 \\
& $(0.01655)$ & $(0.01787)$ & $(0.01748)$ \\
Crecimiento PEA & $1.3168^{* * *}$ & $1.321^{* *}$ & $1.1322^{* *}$ \\
& $(0.48639)$ & $(0.5461)$ & $(0.5183)$ \\
Crecimiento de la FBK & $0.1286^{* * *}$ & $0.1312^{* * *}$ & $0.1346^{* * *}$ \\
Tasa de variación de delitos contra la propiedad & $(0.03542)$ & $(0.03853)$ & $(0.0385)$ \\
& 0.0288 & & \\
Tasa de variación de homicidios & $(0.02411)$ & & \\
Tasa de variación de secuestros & & -0.0094 & \\
& & & \\
& & & $-0.0239)$ \\
\end{tabular}




\begin{tabular}{lccc}
\hline R-cuadrado & 0.4039 & 0.3843 & 0.3909 \\
Errores estándar regresión & 0.01737 & 0.0177 & 0.01755 \\
Criterio de Akaike & -5.1899 & -5.1574 & -5.1683 \\
Observaciones & 49 & 49 & 49 \\
\hline Significancia: *significativo al 10\%, ** significativo al 5\%, $\mathrm{y} * *$ significativo al $1 \%$ & & \\
\hline
\end{tabular}

En la Tabla 1, se observa la estimación de tres regresiones, considerando las diferentes variables de crimen y conflicto. Las variables macroeconómicas, como lo son la tasa de crecimiento anual de la población económicamente activa (PEA) y la tasa de crecimiento anual de la formación bruta de capital fijo (FBKF) tienen un efecto positivo sobre la tasa de crecimiento anual del PIB, confirmando así el planteamiento teórico de los modelos de crecimiento, específicamente el de Solow, que es el base para construir el modelo.

Para las variables proxys de violencia y criminalidad se obtiene un resultado sorprendente, dado que la tasa de crecimiento anual de delitos contra la propiedad y la tasa de crecimiento anual de homicidios no son estadísticamente significativos. Sin embargo, la tasa de crecimiento anual de secuestros sí es significativa y con efecto negativo, confirmando parte de los planteamientos teóricos de la violencia y la criminalidad en la dinámica económica.

Frente al valor de los coeficientes de las regresiones, se recuerda que cada uno mide el efecto promedio de esa variable sobre el crecimiento anual del PIB para el periodo de estudio, manteniendo las demás variables constantes. Por lo que por un aumento en un 1\% de la PEA, el PIB se va a ver aumentado en un 1.3\%. Un aumento en un 1\% de la FBK el PIB se va a ver aumentado en un $0.13 \%$. Ahora, cuando los secuestros aumentan en $1 \%$ el PIB se estima que disminuya su crecimiento en $0.06 \%$.

La única variable de las proxys de violencia y criminalidad que explica el efecto negativo es la tasa de secuestros. Este resultado se puede explicar en el sentido de que el crecimiento económico se ve afectado por una fuga de capitales o por un desanimo a realizar inversiones. A razón de que los agentes con capital disponible se sienten amenazados, se verán obligados a destinar parte de sus recursos para protección y seguridad, siendo éstas unas actividades que les representan unos costos adicionales; por lo que optan por salir del país, mantener sus recursos en activos financieros de bajo riesgo, y los extranjeros no 
traen sus capitales. En definitiva, los resultados son coherentes con los obtenidos en los estudios mencionados, y muestran que la variable mediante la cual se transmite los efectos negativos de la violencia en el crecimiento económico es la tasa de secuestros.

\section{Conclusiones}

Identificar la relación entre violencia y crecimiento económico en Colombia, permitió concluir desde una perspectiva teórica en primer lugar, que el concepto sobre la violencia varía en razón del análisis y orientación que amerita realizarse, en tal sentido la existencia y diversidad de conceptos sobre este fenómeno fortalece el desarrollo del estudio, así como afianza el debate sobre el tema. Desde la perspectiva teórica, se refleja que un hecho conflictivo no necesariamente significa que sea violento o que haya violencia. La diferenciación de conceptos, contribuye a concebir que los actos violentos implican que se cometan una serie de crímenes, pero hay que tener en cuenta que muchos actos criminales no están relacionados con la violencia. Esto a razón de que la criminalidad está sujeta a los conceptos normativos y jurídicos del Estado, y dentro de este se pueden considerar diversas acciones humanas como delitos.

Desde un punto de vista de los estudios sobre la violencia, a través del artículo no se expresa que se deban descartar los estudios tradicionales, debido que estos ofrecen un diagnóstico de la realidad del país desde un punto de vista histórico. De igual manera, sucede con aquellas críticas mal fundamentadas, como la de Sánchez y Núñez (2001) sobre la exclusión política; esto se debe a que el referido trabajo recurre a metodologías más rigurosas que en los tradicionales no se observan, como la de proponer un mayor rigor sobre el estudio estadístico de las variables relacionadas con la violencia, como la pobreza.

En el análisis empírico, se explica la relación entre el crecimiento económico y la violencia, mediante la cual se evidencian limitaciones al momento de desarrollar el análisis estadístico, debido a las dificultades para medir los hechos violentos y criminales (es necesario acudir a proxys). Según las regresiones realizadas se señala que las variables proxy de violencia, como son los secuestros, inciden negativamente en el crecimiento económico, por lo que se comprueba la relevancia de este indicador de crimen para las decisiones de los hacedores de política económica. 
Como aspecto concluyente, se evidencia que la violencia y la criminalidad afectan el desarrollo económico, ya sea desde la perspectiva individual o colectiva, en consecuencia, se esbozan los costes que los fenómenos estudiados pueden tener sobre la economía de manera directa o indirecta. Finalmente, se evidencia la necesidad de seguir realizando más estudios de este tipo, incluyendo el papel de la violencia y las instituciones en el desarrollo económico regional.

\section{Referencias}

Bonilla Meía, L. (2009). Revisión de la literatura económica reciente sobre las causas de la violencia homicida en Colombia. Documentos de Trabajo Sobre Economía Regional , 1-39. Obtenido de http://www.banrep.gov.co/sites/default/files/publicaciones/archivos/DTSER-114.pdf

Echeverry, J. C., Salazar, N., \& Navas, V. (2001). El conflicto colombiano en el marco internacional. En A. Martínez Ortiz (Ed.), Economía, crimen y conflicto (págs. 77-128). Bogotá: Ediciones Antropos LTDA.

Esquivel Guerrero, J. A., Jiménez Bautista, F., \& Esquivel-Sánchez, J. A. (2009). La relación entre conflictos y poder. Revista de Paz y Conflictos , 6-22.

Estrada Gallego, F. (2009). Evolución estratégica del conflcito armado en Colombia. Análisis Político, 156-181. Obtenido de http://www.scielo.org.co/pdf/anpol/ v22n67/v22n67a08.pdf

Gaitán Daza, F. (2001). Multicasualidad,impunidad y violencia: Una visión alternativa. Revista de Economía Institucional, 78-105. Obtenido de http://www.economiainstitucional.com/pdf/No5/fgaitan5.pdf

Gómez, C. M. (2001). Economía y violencia en Colombia. En A. Martínez Ortiz (Ed.), Economía, crimen y conflicto (págs. 41-58). Bogotá: Ediciones Antropos LTDA.

González Andrade, S. (2014). Criminalidad y crecimiento económico regional en México. Revista Frontera Norte, 75-111. 
Granada, S., Restrepo, J. A., \& Sánchez Meertens, C. (2009). Controlando la medición: alcances y limitaciones de la información en conflictos armados. En J. Restrepo, \& D. Aponte (Edits.), Guerra y violencias en Colombia : herramientas e interpretaciones (págs. 203-232). Pontificia Universidad Javeriana. Obtenido de http://www.cerac.org.co/assets/files/guerrayviolencias/3_Controlando_la_medicion.pdf

Guzman Campos, G., Fals Borda, O., \& Umaña Luna, E. (2014). La Violencia en Colombia. Bogotá: Punto de Lectura.

Hofstetter Gascón, M. (1998). La Violencia en los Modelos de Crecimiento Económico. Revista de Economía de la Universidad del Rosario, 67-77. Obtenido de http:// www.urosario.edu.co/urosario_files/ce/ce97c82e-4508-4402-b5d7-7212d85ccedf. pdf

Molina, L. A., \& Hurtado Rendón, Á. (2012). Inestabilidad Institucional, evidencia para Colombia: La violencia y el crecimiento económico en el périodo 1950-2010. Documentos de Trabajo Economía y Finanzas, 1-32. Recuperado el 14 de Abril de 2015, de http://www.eafit.edu.co/escuelas/economiayfinanzas/publicaciones/ Documents/working-papers/Working\%202\%20-\%20Alvaro.pdf

Montenegro, A., \& Posada, C. E. (2001). La Violencia en Colombia. Bogotá: Alfaomega.

Organización Mundial de la Salud. (2003). Informe mundial sobre la violencia y la salud. Washington, D.C: Organización Panamericana de la Salud, Oficina Regional para las Américas de la Organización Mundial de la Salud. Obtenido de http://whqlibdoc.who.int/publications/2003/9275315884_spa.pdf

Rubio, M. (1997). Los costos de la violencia en Colombia. Centro de Estudios Sobre el Desarrollo Económico, 1-23. Obtenido de http://pdba.georgetown.edu/Security/ citizensecurity/Colombia/evaluaciones/costosviolencia.pdf

Rubio, M. (1998). Crimen Con Misterio: La calidad de la información sobre criminalidad y violencia en Colombia. Centro de Estudios Sobre el Desarrollo Eco- 
nómico, 1-37. Obtenido de http://res.uniandes.edu.co/pdf/descargar.php?f=./data/ Revista_No_01/05_Dossier3.pdf

Salas Salazar, L. G. (2010). Corredores y territorios estratégicos del conflicto armado colombiano: una prioridad por territorializar en la geopolítica de los actores armados. Perspectiva Geográfica, 9-36.

Salas Salazar, L. G. (2015). Lógicas territoriales y relaciones de poder en el espacio de los actores armados: un aporte desde la geografía política al estudio de la violencia y el conflicto armado en Colombia, 1990-2012. Cuadernos de Geografía: Revista Colombiana de Geografía, 157 - 172.

Sánchez Torres, F., \& Núñez Méndez, J. (2001). Determinantes del Crimen Violento en un País Alatamente Violento: El Caso de Colombia. Centro de Estudios Sobre el Desarrollo Económico, 1-45. Obtenido de http://issuu.com/carlosgutierrez30/ docs/determinantes__del__crimen__violent

Sánchez, F., Díaz, A. M., \& Formisano, M. (2003). Conflcito, violencia y actividad criminal en Colombia: Un análisis espacial. Centro de Estudios Sobre el Desarrollo Económico, 1-60.

Santa María Salamanca, M., Rojas Delgadillo, N., \& Hernández Díaz, G. (2013). Crecimiento económico y Conflicto Armado en Colombia. Archivos de Economía, 1-13. Obtenido de https://colaboracion.dnp.gov.co/CDT/Estudios\%20Econmicos/400.pdf

Tobar Torres, J. (2015). Violencia política y guerra sucia en Colombia. Memoria de una víctima del conflicto colombiano a propósito de las negociaciones de la Habana. Memoria y Sociedad, 9-22.

Villa , E., Moscoso, M., \& Restrepo, J. (2013). Crecimiento, conflicto armado y crimen organizado: Evidencia para Colombia. Vniversitas Económica, 1-26. Obtenido de http://cea.javeriana.edu.co/documents/153049/2786252/Vol.13_3_2013. pdf/70798ac6-81ab-4b2d-bba7-231f1d3fb43b 